\title{
ABSTRACT FROM CURRENT LITERATURE
}

Efficacy of recommended drugs against soil transmitted helminths: systematic review and network meta analysis

Moser W, Schindler C, Keiser J, et al

BMJ 2017; 358:j4307

Objective: To evaluate efficacies of anthelmintic drugs against soil transmitted helminths in terms of cure rates and egg reduction rates.

Design: Systematic review and network meta analysis.

Data Sources: PubMed, ISI Web of Science, Embase, ScienceDirect, the Cochrane Central Register of Clinical Trials, and the World Health Organization library database from 1960 until 31 December 2016.

Study selection: Randomised controlled trials evaluating the efficacy of a single dose regimen of albendazole, mebendazole, levamisole, and pyrantel pamoate against Ascaris lumbricoides, hookworm (Necator americanus and Ancylostoma duodenale) and Trichuris trichiura. The primary outcomes included cure rates analysed by network meta analysis with mixed logistic regression models and egg reduction rates with mixed linear models.

Results: 55 and 46 randomised controlled trials were included in the analysis of cure rates and egg reduction rates, respectively. All drugs were highly efficacious against A lumbricoides. Albendazole showed the highest efficacy against hookworm infections with a cure rate of $79.5 \% \quad(95 \%$ confidence interval $71.5 \%$ to $85.6 \%$ ) and an egg reduction rate of $89.6 \%(81.9 \%$ to $97.3 \%)$. All drugs had low efficacy against $\mathrm{T}$ trichiura, with mebendazole showing the highest cure rate of $42.1 \%(25.9 \%$ to $60.2 \%)$ and egg reduction rate of $66.0 \%$ (54.6\% to $77.3 \%$ ). Estimates for the years 1995 and 2015 showed significant reductions in efficacy of albendazole against $\mathrm{T}$ trichiura: by 2015 the egg reduction rates fell from $72.6 \%$ $(53.7 \%$ to $91.5 \%)$ to $43.4 \%(23.5 \%$ to $63.3 \%$; $\mathrm{P}$ $0.049)$ and the cure rates fell from $38.6 \%(26.2 \%$ to $52.7 \%)$ to $16.4(7.7 \%$ to $31.3 \%$; $\mathrm{P} 0.027)$.

Conclusions: All four currently recommended drugs show limitations in their efficacy profile. While only albendazole showed good efficacy against hookworm infection, all drugs had low efficacy against $T$ trichiura. The decrease in efficacy of albendazole against $\mathrm{T}$ trichiura over the past two decades is of concern. The findings indicate the need for strengthening efforts to develop new drug treatments, with a particular focus on drugs against $\mathrm{T}$ trichiura.
Long term gluten consumption in adults without celiac disease and risk of coronary heart disease: prospective cohort study

Lebwohl B, Cao Y, Zong G, et al

BMJ 2017; 357:j1892

Objective: To examine the association of long term intake of gluten with the development of incident coronary heart disease.

Design: Prospective cohort study.

Setting and participants: 64714 women in the Nurses' Health Study and 45303 men in the Health Professionals Follow up Study without a history of coronary heart disease who completed a 131 item semiquantitative food frequency questionnaire in 1986 that was updated every four years through 2010 .

Exposure: Consumption of gluten, estimated from food frequency questionnaires.

Main outcome measure: Development of coronary heart disease (fatal or non fatal myocardial infarction).

Results: During 26 years of follow up encompassing 2273931 person years, 2431 women and 4098 men developed coronary heart disease. Compared with participants in the lowest fifth of gluten intake, who had a coronary heart disease incidence rate of 352 per 100000 person years, those in the highest fifth had a rate of 277 events per 100000 person years, leading to an unadjusted rate difference of 75 (95\% confidence interval 51 to 98) fewer cases of coronary heart disease per 100000 person years. After adjustment for known risk factors, participants in the highest fifth of estimated gluten intake had a multivariable hazard ratio for coronary heart disease of 0.95 (95\% confidence interval 0.88 to 1.02; $\mathrm{P}$ for trend=0.29). After additional adjustment for intake of whole grains (leaving the remaining variance of gluten corresponding to refined grains), the multivariate hazard ratio was 1.00 (0.92 to 1.09; P for trend=0.77). In contrast, after additional adjustment for intake of refined grains (leaving the variance of gluten intake correlating with whole grain intake), estimated gluten consumption was associated with a lower risk of coronary heart disease (multivariate hazard ratio 0.85, 0.77 to 0.93; $\mathrm{P}$ for trend=0.002).

Conclusion: Long term dietary intake of gluten was not associated with risk of coronary heart disease. However, the avoidance of gluten may result in reduced consumption of beneficial whole grains, which may affect cardiovascular risk. The promotion of gluten free diets among people without celiac disease should not be encouraged. 
Effectiveness of non steroidal anti inflammatory drugs for the treatment of pain in knee and hip osteoarthritis: a network meta analysis

Costa BR, Reichenbach $S$, Keller N, et al

The Lancet; 2017; 390, 10090, e2l-e33

Background: Non steroidal anti inflammatory drugs (NSAIDs) are the backbone of osteoarthritis pain management. We aimed to assess the effectiveness of different preparations and doses of NSAIDs on osteoarthritis pain in a network meta analysis.

Methods: For this network meta analysis, we considered randomised trials comparing any of the following interventions: NSAIDs, paracetamol, or placebo, for the treatment of osteoarthritis pain. We searched the Cochrane Central Register of Controlled Trials (CENTRAL) and the reference lists of relevant articles for trials published between Jan 1, 1980, and Feb 24, 2015, with at least 100 patients per group. The prespecified primary and secondary outcomes were pain and physical function, and were extracted in duplicate for up to seven timepoints after the start of treatment. We used an extension of multivariable Bayesian random effects models for mixed multiple treatment comparisons with a random effect at the level of trials. For the primary analysis, a random walk of first order was used to account for multiple follow up outcome data within a trial. Preparations that used different total daily dose were considered separately in the analysis. To assess a potential dose response relation, we used preparation specific covariates assuming linearity on log relative dose.

Findings: We identified 8973 manuscripts from our search, of which 76 randomised trials with a total of 58451 patients were included in this analysis. 23 nodes concerning seven different NSAIDs or paracetamol with specific daily dose of administration or placebo were considered. All preparations, irrespective of dose, improved point estimates of pain symptoms when compared with placebo. For six interventions (diclofenac 150 $\mathrm{mg} /$ day, etoricoxib $30 \mathrm{mg} /$ day, $60 \mathrm{mg} /$ day, and $90 \mathrm{mg} /$ day, and rofecoxib $25 \mathrm{mg} /$ day and 50 $\mathrm{mg} /$ day), the probability that the difference to placebo is at or below a prespecified minimum clinically important effect for pain reduction (effect size [ES] -0.37) was at least 95\%. Among maximally approved daily doses, diclofenac 150 $\mathrm{mg} /$ day (ES -0.57, 95\% credibility interval [Cril] 0.69 to -0.45 ) and etoricoxib $60 \mathrm{mg} /$ day (ES $0.58,-0.74$ to -0.43 ) had the highest probability to be the best intervention, both with $100 \%$ probability to reach the minimum clinically important difference. Treatment effects increased as drug dose increased, but corresponding tests for a linear dose effect were significant only for naproxen $(p=0.034)$. We found no evidence that treatment effects varied over the duration of treatment. Model fit was good, and between trial heterogeneity and inconsistency were low in all analyses. All trials were deemed to have a low risk of bias for blinding of patients. Effect estimates did not change in sensitivity analyses with two additional statistical models and accounting for methodological quality criteria in meta regression analysis.

Interpretation: On the basis of the available data, we see no role for single agent paracetamol for the treatment of patient \& with osteoarthritis irrespective of dose. We provide sound evidence that diclofenac $150 \mathrm{mg} /$ day is the most effective NSAID available at present, in terms of improving both pain and function. Nevertheless, in view of the safety profile of these drugs, physicians need to consider our results together with all known safety information when selecting the preparation and dose for individual patients.

\section{Effect of azithromycin on asthma exacerbations and quality of life in adults with persistent uncontrolled asthma (AMAZES): a randomised, double blind, placebo controlled trial}

Gibson PG, Yang 1A, Upham J, et al

The Lancet; 2017; 390, 10095, 659668

Background: Exacerbations of asthma cause a substantial global illness burden. Adults with uncontrolled persistent asthma despite maintenance treatment require additional therapy. Since macrolide antibiotics can be used to treat persistent asthma, we aimed to assess the efficacy and safety of oral azithromycin as add on therapy in patients with uncontrolled persistent asthma on medium to high dose inhaled corticosteroids plus a long acting bronchodilator.

Methods: We did a randomised, double blind, placebo controlled parallel group trial to determine whether oral azithromycin decreases the frequency of asthma exacerbations in adults $(>18$ years) with symptomatic asthma despite current use of inhaled corticosteroid and long acting bronchodilator and who had no hearing impairment or abnormal prolongation of the corrected QT interval. Patients were randomly assigned $(1: 1)$ to receive azithromycin $500 \mathrm{mg}$ or placebo three times per week for 48 weeks. Patients were centrally allocated using concealed random allocation from a computer generated random numbers table with permuted blocks of 4 or 6 and stratification for centre and past smoking. Primary efficacy endpoints were the rate of total (severe and moderate) asthma exacerbations over 48 weeks and asthma quality of life. Data were analysed on an intention to treat basis. The trial is registered at the Australian and New Zealand Clinical Trials Registry (ANZCTR), number 12609000197235. 
Findings: Between June 12, 2009, and Jan 31, 2015,420 patients were randomly assigned (213 in the azithromycin group and 207 in the placebo group). Azithromycin reduced asthma exacerbations (1.07 per patient year $[95 \% \mathrm{Cl} 0.85$ 1.29] compared with placebo (1.86 per patient year [1.54-2.181; incidence rate ratio [IRR] 059 [95\% Cl 0.47-0.74]; $\mathrm{p}<0.0001$ ). The proportion of patients experiencing at least one asthma exacerbation was reduced by azithromycin treatment (127 [61\%] patients in the placebo group vs 94 [44\%] patients in the azithromycin group, $\mathrm{p}<0.0001)$. Azithromycin significantly improved asthma related quality of life (adjusted mean difference, 0.36 [95\% C1 0.21-0.521; p=0 .001). Diarrhoea was more common in azithromycin treated patients $(72$ [34\%] vs 39 [19\% $1 ; \mathrm{p}=0$ 001).

Interpretation: Adults with persistent symptomatic asthma experience fewer asthma exacerbations and improved quality of life when treated with oral azithromycin for 48 weeks. Azithromycin might be a useful add on therapy in persistent asthma.

Pseudotumor cerebri syndrome in childhood: incidence, clinical profile and risk factors in a national prospective population based cohort study

Matthews Y-Y, Dean F, Lim MJ, et al

Archives of Disease in Childhood 2017; 102:715-721.

Aim: To investigate the epidemiology, clinical profile and risk factors of pseudotumor cerebri syndrome (PTCS) in children aged 1-16 years.

Methods: A national prospective population based cohort study over 25 months. Newly diagnosed PTCS cases notified via British Paediatric Surveillance Unit were ascertained using classical diagnostic criteria and categorised according to 2013 revised diagnostic criteria. We derived national age, sex and weight specific annual incidence rates and assessed effects of sex and weight categories.

Results: We identified 185 PTCS cases of which 166 also fulfilled revised diagnostic criteria. The national annual incidence $(95 \% \mathrm{CI})$ of PTCS in children aged $1-16$ years was 0.71 (0.57 to 0.87 ) per 100000 population increasing with age and weight to 4.18 and 10.7 per 100000 in obese boys and girls aged 12-15 years, respectively. Incidence rates under 7 years were similar in both sexes. From 7 years onwards, the incidence in girls was double that in boys, but only in overweight (including obese) children. In children aged 12-15 years, an estimated $82 \%$ of the incidence of PTCS was attributable to obesity. Two subgroups of PTCS were apparent: 168 (91\%) cases aged from 7 years frequently presented on medication and with headache and were predominantly female and obese. The remaining $17(9 \%)$ cases under 7 years often lacked these risk factors and commonly presented with paralytic squint.
Conclusions: This uniquely large population based study of childhood PTCS will inform the design of future intervention studies. It suggests that weight reduction is central to the prevention of PTCS.

Screen time is associated with adiposity and insulin resistance in children

Nightingale CM, Rudnicka AR, Donin AS, et al

Archives of Disease in Childhood 2017;102:612-616.

Background: Higher screen time is associated with type 2 diabetes (T2D) risk in adults, but the association with T2D risk markers in children is unclear. We examined associations between self reported screen time and $\mathrm{T} 2 \mathrm{D}$ risk markers in children.

Methods: Survey of 4495 children aged 9-10 years who had fasting cardiometabolic risk marker assessments, anthropometry measurements and reported daily screen time; objective physical activity was measured in a subset of 2031 children.

Results: Compared with an hour or less screen time daily, those reporting screen time over 3 hours had higher ponderal index $(1.9 \%, 95 \% \mathrm{Cl}$ $0.5 \%$ to $3.4 \%)$, skinfold thickness $(4.5 \%, 0.2 \%$ to $8.8 \%)$, fat mass index $(3.3 \%, 0.0 \%$ to $6.7 \%)$, leptin $(9.2 \%, 1.1 \%$ to $18.0 \%)$ and insulin resistance $(10.5 \%, 4.9 \%$ to $16.4 \%)$; associations with glucose, $\mathrm{HbA}$, physical activity and cardiovascular risk markers were weak or absent. Associations with insulin resistance remained after adjustment for adiposity, socioeconomic markers and physical activity.

Conclusions: Strong graded associations between screen time, adiposity and insulin resistance suggest that reducing screen time could facilitate early T2D prevention. While these observations are of considerable public health interest, evidence from randomised controlled trials is needed to suggest causality.

\section{Percutaneous cholecystostomy in the management of high risk patients presenting with acute cholecystitis: Timing and outcome at a single institution}

Zarour S, Imam A, Kouniavsky G, et al

The American Journal of Surgery 2017; 214, 456-461

Background: Cholecystectomy is the standard of care in acute cholecystitis (AC). Percutaneous cholecystostomy (PC) is an effective alternative for high risk surgical cases.

Methods: A retrospective analysis is presented of AC patients treated with PC drainage at a single tertiary institution over a 21 month period, assessing outcome and complications.

Results: Of 119 patients, 103 had clinical improvement after PC insertion. There were 7 peri procedural deaths $(5.9 \%)$, all in elderly high risk cases. Overall, 56/103 cases (54\%) were definitively managed with PC drainage with 41 
patients (40\%) undergoing an elective cholecystectomy (75\% performed laparoscopically). The timing of PC insertion did not affect AC resolution or drain related complications, although more patients underwent an elective cholecystectomy if PC placement was delayed ( $>24$ $\mathrm{h}$ after admission).

Conclusions: In AC, drainage by a PC catheter is a safe and effective procedure. It may be used either as a bridge to elective cholecystectomy or in selected cases as definitive therapy.

\section{Partial versus complete removal of the infected mesh after abdominal wall hernia repair}

Bueno-Lledo J, Torregrosa Gallud AT, Carreno Saenz O, et al The American Journal of Surgery 2017; 214, 47-52

Background: To compare the results with complete mesh removal (CMR) versus partial mesh removal (PMR) in the treatment of mesh infection after abdominal wall hernia repair (AWHR).

Methods: Retrospective review of all patients who underwent surgery for mesh infection between January 2004 and May 2014 at a tertiary center.

Results: Of 3470 cases of AWHR, we reported 66 cases $(1.9 \%)$ of mesh infection, and 48 repairs $(72.7 \%)$ required mesh explantation. CMR was achieved on 38 occasions, while PMR was undertaken ten times. We observed more postoperative complications in CMR than PMR group $(\mathrm{p}=0.04)$. Three patients with intestinal fistula were reoperated in postoperative period after a difficult mesh removal; one of them died due to multiple organ failure. The overall recurrence rate after explantation was $47.9 \%$ : recurrence was more frequent in CMR group $(\mathrm{p}=$ $0.001)$, although persistent or new mesh infection was observed more frequently with PMR $(p=0.001)$.

Conclusions: Although PMR has less postoperative morbidity, shorter duration of hospitalization and lower rate of recurrence than $\mathrm{CMR}$, prosthetic infection persists in up to $50 \%$ of cases.

\section{Risk of complication during surgical abortion in obese women}

Mark KS, Bragg B, Talaie T, et al

American J Obs. \& Gyne DOI. j.ajog.2017; 10.018

Objectives: To determine if obesity is a risk factor for major complications in surgical abortions.

Methods: A quality control database from a single outpatient center was analyzed to determine rates of major complications during surgical abortions in relation to obesity class. Complications included hemorrhage, need for repeat evacuation, uterine perforation, cervical laceration, medication reaction, unexpected surgery or unplanned admission to the hospital. Chi squared and ANOVA were used for analysis.
Results: 2,468 procedures were included, 1,475 $(59.8 \%)$ in the first trimester and $993(40.2 \%)$ in the second trimester. The overall complications rate was $2.2 \%$. Second trimester procedures were more likely than those in the first trimester to have complications $(3.1 \% \mathrm{v} 1.6 \%, \mathrm{p}=0.009)$. Overall, $39.6 \%$ of women were obese and $9.6 \%$ met criteria for Class 3 obesity (BMI > 40). Women undergoing second trimester abortions with Class 3 obesity had a rate of complications of $8.7 \%$, which was significantly more than normal weight women (OR 5.90, 95\% Cl 1.93-8.07, p < 0.001).

Comment: Surgical abortions are overall safe procedures, but Class 3 obesity increases the rate of complication in second trimester procedures.

\section{Metformin, the aspirin of the 21 st century: its role in gestational diabetes mellitus, prevention of preeclampsia and cancer, and the promotion of longevity}

Romero R, Erez O, Huttemann. $M$, et al.

American J Obs. \& Gynecology 2017; 217: 282-302

Metformin is everywhere. Originally introduced in clinical practice as an antidiabetic agent, its role as a therapeutic agent is expanding to include treatment of prediabetes mellitus, gestational diabetes mellitus, and polycystic ovarian disease; more recently, experimental studies and observations in randomized clinical trials suggest that metformin could have a place in the treatment or prevention of preeclampsia. This article provides a brief overview of the history of metformin in the treatment of diabetes mellitus and reviews the results of metaanalyses of metformin in gestational diabetes mellitus as well as the treatment of obese, non diabetic, pregnant women to prevent macrosomia. We highlight the results of a randomized clinical trial in which metformin administration in early pregnancy did not reduce the frequency of large for gestational age infants (the primary endpoint) but did decrease the frequency of preeclampsia (a secondary endpoint). The mechanisms by which metformin may prevent preeclampsia include a reduction in the production of antiangiogenic factors (soluble vascular endothelial growth factor receptor-I and soluble endoglin) and the improvement of endothelial dysfunction, probably through an effect on the mitochondria. Another potential mechanism whereby metformin may play a role in the prevention of preeclampsia. is its ability to modify cellular homeostasis and energy disposition, mediated by rapamycin, a mechanistic target. Metformin has a molecular weight of 129 Daltons and therefore readily crosses the placenta. There is considerable evidence to suggest that this agent is safe during pregnancy. New literature on the role of metformin as a chemotherapeutic adjuvant in the prevention of cancer and in prolonging life and protecting against aging is reviewed briefly. Herein, we discuss the mechanisms of action and potential benefits of metformin. 